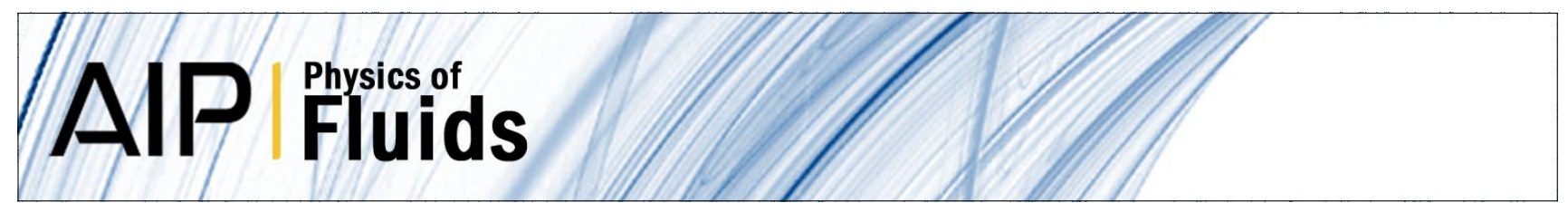

\title{
Temperature and entropy in supersonic free jets
}

\author{
S. Montero
}

Citation: Phys. Fluids 25, 056102 (2013); doi: 10.1063/1.4807160

View online: http://dx.doi.org/10.1063/1.4807160

View Table of Contents: http://pof.aip.org/resource/1/PHFLE6/v25/i5

Published by the American Institute of Physics.

\section{Additional information on Phys. Fluids}

Journal Homepage: http://pof.aip.org/

Journal Information: http://pof.aip.org/about/about_the_journal

Top downloads: http://pof.aip.org/features/most_downloaded

Information for Authors: http://pof.aip.org/authors

\section{ADVERTISEMENT}

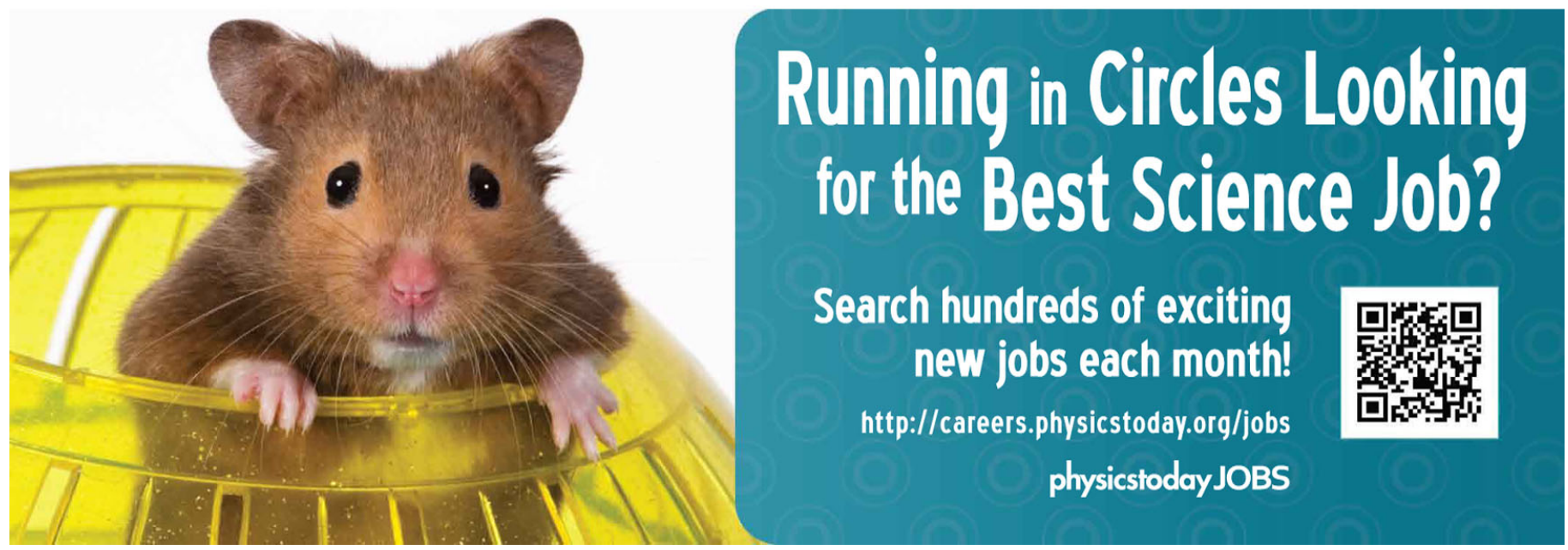


PHYSICS OF FLUIDS 25, 056102 (2013)

\title{
Temperature and entropy in supersonic free jets
}

\author{
S. Montero ${ }^{a)}$ \\ Laboratory of Molecular Fluid Dynamics, Instituto de Estructura de la Materia, \\ CSIC Serrano 121, 28006 Madrid, Spain
}

(Received 25 January 2013; accepted 1 May 2013; published online 29 May 2013)

\begin{abstract}
Translational temperature $\left(T_{t}\right)$, flow velocity $(v)$, and entropy increment $(\Delta S)$ along the paraxial zone of silence of supersonic free jets are studied in the frame of the Navier-Stokes (NS) equations. Expressions for the experimentally non-accessible quantities $T_{t}, v$, and $\Delta S$ are formulated in terms of the experimentally accessible number density $(n)$ and rotational temperature $\left(T_{r}\right)$. The scope of the inviscid-adiabatic and isentropic flow approximations is discussed with emphasis in the dissipative effects onto the flow variables. The influence of dissipative contributions due to viscosity and heat conductivity onto the translational temperature and entropy of pure helium supersonic jets is illustrated. Two sources of entropy increment in the jets have been identified and formulated quantitatively, namely, the dissipative effects, and the $T_{r} \neq T_{t}$ non-equilibrium in gases including molecular species. As far as the rarefaction of the jet allows for the use of the NS-equations, a number of "exact" expressions relating the flow variables $n, T_{t}, T_{r}, v$, and $\Delta S$ along mixed supersonic jets of atoms and molecules are reported. These expressions are aimed at an optimal experimental diagnostics of the jets. (c)2013 AIP Publishing LLC. [http://dx.doi.org/10.1063/1.4807160]
\end{abstract}

\section{INTRODUCTION}

Supersonic free jets underlie a number of technical and scientific applications. Among their remarkable properties is the extremely high cooling efficiency along the expansion, which may reach rates up to $10^{10} \mathrm{~K} / \mathrm{s}$. Moreover, the expanded gas may remain uncondensed up to very low temperature, in a non-equilibrium state characterized by different temperatures for the translational, rotational, and vibrational degrees of freedom. This breakdown of equilibrium, which is progressive along the expansion in the so called "zone of silence," provides a unique medium for the experimental study of inelastic collisions in cold gases. ${ }^{1-8}$ Such studies are quantitative in nature and demand, however, an accurate characterization of the local flow variables, usually the number density $n$, the rotational populations $P_{i}$ associated with the quantum states $i$, the translational and rotational temperatures, $T_{t}$ and $T_{r}$, respectively, and the flow velocity $v$. While $n, P_{i}$, and $T_{r}$ can be measured today with very good accuracy by means of Raman spectroscopy with high space resolution ${ }^{9-15}$ as well as by other methods, ${ }^{16-18}$ translational temperatures cannot be measured routinely along the zone of silence, at least within the first 100 nozzle diameters of the jet. This is the region of interest for the study of collisions.

Accurate measurement of the translational temperature $T_{t}$ along the jet, from close to the nozzle up to the normal shock wave is a major experimental challenge. Interferometry of Doppler profiles of spectral lines from laser induced fluorescence in hypersonic wind tunnel experiments has shown the feasibility of accurate $T_{t}$ measurements in pure helium jets. ${ }^{19,20}$ However, no further systematic measurements of $T_{t}$ on molecular jets have been reported as far as the author is aware. Promising methods based in Rayleigh scattering measurements appear to be in a developmental stage. ${ }^{21,22}$ Terminal translational temperatures that can be measured in molecular beams far downstream in

\footnotetext{
a)Electronic mail: emsalvador@iem.cfmac.csic.es
} 
the jet by time of flight methods ${ }^{23,24}$ provide little information on the evolution of the translational temperature in the zone of interest for the study of collisions. The first goal of present work is to overcome these limitations, providing equations which allow for an indirect determination of $T_{t}$ on the basis of conservation principles (mass, linear momentum, and energy) in the frame of the Navier-Stokes equations, and of the experimentally accessible quantities $n$ and $T_{r}$.

In many works dealing with supersonic jets it is implicitly or explicitly accepted that the flow along the paraxial region of supersonic jets is isentropic. Moreover, it is also assumed that the inviscid-adiabatic approximation is equivalent to the isentropic one. These assumptions, which may be a good approximation in some cases, are not true in general. Approximations based on the presumed isentropic behavior of the jet will be discussed below. On the other hand viscous and diabatic effects onto temperature and entropy along the paraxial supersonic flow have been considered before in one or other way. ${ }^{25-28}$ These results are, however, difficult to apply in practice to the present state of experimental diagnostics.

Since the flow variables $n, T_{r}$, and $T_{t}$ in jets may vary by orders of magnitude depending on the stagnation conditions, nature of the expanded gas, and distance to the nozzle, it is not easy to conclude a priori about the increment of entropy $\Delta S$ along a particular jet. Earlier attempts to account for $\Delta S$ in terms of power series of Reynolds $(R e)$ and Mach number $(M)^{25}$ apparently have not been of practical significance. Hence, a rigorous discussion of the evolution of entropy along the jet is pertinent as the second main goal of this work.

\section{FROM NAVIER-STOKES TO JET EQUATIONS}

The jet whose paraxial region is studied next will be treated as the expansion of a real gas through an ideal nozzle. The gas is assumed to display shear viscosity $\mu$, volume viscosity $\eta_{V}$, and thermal conductivity $\lambda$, quantities which depend on temperature. The molecules in the expanding gas are supposed to interchange energy only by means of elastic and inelastic collisions, excluding reactive and cohesive collisions, not emitting nor absorbing radiation.

As an ideal nozzle it is meant here an orifice of undetermined shape (circular, rectangular slit, or any other shape) in an infinite surface of infinitesimal width. This surface separates the stagnation chamber from the expansion chamber, both chambers considered of infinite volume. The expansion chamber is supposed to be in perfect vacuum, thus excluding the formation of normal and barrel shocks in the jet. The gas in the jet is assumed to expand steadily, condition which implies that the ensemble of molecules in a space-fixed differential volume is continuously replenished in time by other ensembles of identical macroscopic properties.

The paraxial region of the jet is referred to the $z$ axis, which is normal to the plane of the ideal nozzle $(z=0$ plane). This plane is taken as the origin of distances. However, this does not mean that the expansion starts there, but at an undetermined point $z_{0}<0$ upstream to the orifice, where the flow velocity $v_{0}$ can be considered zero, with the gas in thermodynamic equilibrium at the stagnation temperature and pressure, $T_{0}$ and $p_{0}$, respectively.

The orifice plane $(z=0)$ separates two different gas-dynamic regimes, the subsonic one with Mach number $M<1$ for $z<0$, and the supersonic one with $M>1$ for $z>0$. At $z=0$, the flow velocity equals the velocity of sound $(a)$ at the local temperature (which should not be confused with the stagnation temperature $T_{0}$ ), becoming $M_{z=0}=1$ for an ideal nozzle.

For a gas under steady expansion not subject to external fields the NS-equations in Cartesian coordinates $\mathrm{read}^{29}$

$$
\begin{aligned}
& \frac{\partial}{\partial x_{j}} \quad\left(\rho u_{j}\right)=0, \\
& \frac{\partial}{\partial x_{j}}\left(\rho u_{i} u_{j}+\delta_{i j} p\right)=d_{1}, \\
& \frac{\partial}{\partial x_{j}}\left(u_{j}\left(\rho e_{t o t}+p\right)\right)=d_{2},
\end{aligned}
$$


where $\rho$ is the density, which will be expressed for later convenience in terms of the molecular mass $m$ (in $\mathrm{kg}$ ) and the number density $n$ (in $\mathrm{m}^{-3}$ ), or in terms of the molar mass $W$ (in $\mathrm{kg} / \mathrm{mol}$ ) and the (molar) number density $\mathcal{N}\left(\right.$ in $\mathrm{mol} \mathrm{m}^{-3}$ ) as

$$
\rho=m n=W \mathcal{N} .
$$

Here $u_{j}$ (in $\mathrm{m} / \mathrm{s}$ ) are the components of the flow velocity vector $\mathbf{v}=\left(u_{x}, u_{y}, u_{z}\right)$ of modulus $|\mathbf{v}|=v$. No slip effects between species in a gas mixture will be considered, $v$ referring to the bulk flow velocity of the gas; $\delta_{i j}$ is Kronecker tensor, and

$$
p=n k_{B} T_{t}=\mathcal{N} R T_{t}
$$

is the pressure (in Pa); $T_{t}$ is the average of parallel and perpendicular translational temperatures; $k_{B}$ is Boltzmann constant, and $R=8.31451 \mathrm{JK}^{-1} \mathrm{~mol}^{-1}$ the universal gas constant. $T_{t}$ accounts for the mean kinetic energy of the molecular motion in the flow. The use of $T_{t}$ in the discussion below allows for a relatively simple set of flow equations, but imposes some limitations in the far downstream section of the jets, usually at distances larger than 50 nozzle diameters. From there on, the separation of the perpendicular and parallel components of the translational temperature may be large enough to invalidate the average temperature approximation. In this case, additional moment equations are needed, as has been discussed by several authors. ${ }^{27,28,30}$

The total energy of the gas per mass unit is

$$
e_{t o t}=e_{i n t}+v^{2} / 2
$$

(in $\mathrm{J} / \mathrm{kg}$ ), where $e_{\text {int }}$ is the energy per mass unit of the random motion of the molecules. This includes the three translational degrees of freedom, and the rotational and vibrational degrees of freedom; $v^{2} / 2$ is the directional kinetic energy of the flow per mass unit; $E_{t o t}=W e_{t o t}$ and $E_{\text {int }}=W e_{\text {int }}$ are, respectively, the the total energy and the internal energy per mol (in J/mol). They are related by

$$
E_{t o t}=E_{i n t}+W v^{2} / 2=\frac{3}{2} R T_{t}+E_{r}+E_{v}+W v^{2} / 2,
$$

where $E_{r}$ and $E_{v}$ are the rotational and vibrational energies per mol; $E_{r}$ and $E_{v}$ are usually expressed in terms of the rotational and vibrational temperatures, $T_{r}$ and $T_{v}$, respectively. The pressure term in Eq. (1) can be expressed as

$$
\left(\rho e_{t o t}+p\right)=\mathcal{N}\left(H+W \frac{v^{2}}{2}\right)
$$

where

$$
H=\frac{5}{2} R T_{t}+E_{r}+E_{v}
$$

is the (molar) enthalpy.

The right-hand terms

$$
\begin{gathered}
d_{1}=\frac{\partial}{\partial x_{j}} \tau_{i j}, \\
d_{2}=\frac{\partial}{\partial x_{j}}\left(u_{\ell} \tau_{\ell j}+\lambda \partial T / \partial x_{j}\right)
\end{gathered}
$$

in Eq. (1) are dissipative and depend on the deviatoric tensor ${ }^{29,31,32}$

$$
\tau_{i j}=\left(\mu+\frac{3}{4} \eta_{V}\right)\left(\frac{\partial u_{i}}{\partial x_{j}}+\frac{\partial u_{j}}{\partial x_{i}}-\frac{2}{3} \delta_{i j} \frac{\partial u_{\ell}}{\partial x_{\ell}}\right) .
$$

The range of validity of the results reported below is determined by the rarefaction of the expanded gas along the jet. This is usually characterized by the local Knudsen number defined as ${ }^{33}$

$$
K n(z)=\frac{\Lambda}{\mathcal{N}}\left|\frac{d \mathcal{N}}{d z}\right|
$$


where

$$
\Lambda=\left(\sqrt{2} N_{\mathrm{Av}} \mathcal{N} \pi d^{2}\right)^{-1}
$$

is the mean free path for a rigid sphere molecule of collision diameter $d$; $N_{\mathrm{Av}}$ is Avogadro's constant.

In the supersonic free jets considered here all four flow regimes appear sequentially, (i) continuum flow, close to the nozzle, with $K n<0.01$, (ii) slip flow, with $0.01<K n<0.1$, (iii) transition flow, with $0.1<K n<1$, and (iv) free molecule flow, with $K n>1$, far downstream in the jet at very high rarefaction. It is widely accepted that conservation equations in the NS-system are valid in the continuum and slip regimes and also in the transition regime up to $K n \approx 0.3{ }^{33,34}$ Beyond this limit, up to $K n \approx 1$, the present results are probably not accurate. In the free molecule regime, with $K n>1$, proper of the far downstream region of the jet beyond 50 nozzle diameters, the present results need further assessment. An approach based on Boltzmann equation should be employed in this case. ${ }^{28}$ However, contradictorily as it may appear with the predicted failure of the fluid equations in transitional hypersonic flows, ${ }^{35}$ numerical NScalculations of stationary supersonic free jet flow properties in this far downstream region show reasonably agreement with the experimental results. ${ }^{30,36}$ This apparent contradiction may be due to the different systems considered, unperturbed free jets expanded into perfect vacuum with no shock front as in the present work, or hypersonic flows with shock front or an immersed body surface. ${ }^{35}$

On the other hand, a fundamental property of all molecular supersonic jets is the $T_{r} \neq T_{t}$ nonequilibrium, which increases with distance $z$ to the nozzle. Since the number density decreases with expansion (approximately as $z^{-2}$ for axisymmetric jets), the difference $T_{r}-T_{t}$ is correlated with rarefaction. It must be emphasized, however, that large $T_{r}-T_{t}$ differences are attained easily in laboratory free jets under modest rarefaction. Examples of $T_{r}-T_{t} \approx 100 \mathrm{~K}$ differences have been reported for $\mathrm{H}_{2}$ jets, ${ }^{3,4,6} T_{r}-T_{t} \approx 60 \mathrm{~K}$ for $\mathrm{H}_{2} \mathrm{O}+\mathrm{He}$ jets, ${ }^{8} T_{r}-T_{t} \approx 20 \mathrm{~K}$ for $\mathrm{O}_{2}$ jets, ${ }^{7}$ and $T_{r}$ $-T_{t} \approx 10 \mathrm{~K}$ for $\mathrm{N}_{2}$ jets, ${ }^{5}$ in all cases at distances between 10 and 50 diameters downstream from the nozzle. These jets and similar jets obeying the $K n<0.3$ rarefaction condition can be expected to be tractable within the present approach.

The present work is not aimed at solving the NS-equations, a specific task for the numerical codes available. Instead, it is intended here to adapt the NS-equations and to complement them with additional equations to the present day experimental state of the art, aimed at retrieving as much experimental information about the jet, and as accurate as possible, in particular on the translational temperature, the flow velocity, and the increment of entropy.

The quantities that can be measured with good accuracy along the jet, from very close to the nozzle up to tens of diameters downstream in the flow are ${ }^{1,3-5,7,9-15,18}$

- $z$, the distance to the nozzle, with accuracy of few $\mu \mathrm{m}$.

- $\mathcal{N}(z)$, the local number density, spanning up to five orders of magnitude, with representative accuracy of about $1 \%$ along the first order of magnitude, $3 \%$ for the second, $10 \%$ for the third, and so on, under optimal conditions.

- $P_{i}(z)$, the local population probabilities of rotational quantum states $i$, subject to the constraint $\sum_{i} P_{i}=1$. Representative accuracy of $P_{i}$ s under routine conditions is 0.001 for diatomic molecules.

- A rotational temperature $T_{r}$ can be obtained from the populations $P_{i}$ of the lowest rotational levels as far as they obey a Boltzmann distribution. Representative accuracy for $T_{r}$ is $1 \mathrm{~K}$.

The one-dimensional approximation of NS-equations is exact for the flow through an infinitesimal axisymmetric stream tube. ${ }^{14,36}$ Since the present day capabilities of high spaceresolution Raman spectroscopy allow sampling the paraxial region of the jet as a narrow stream tube, ${ }^{1-7,11,12,14}$ the paraxial region of the jet can be treated numerically and experimentally in good approximation as a one-dimension problem along $z$ axis, the reduced NS-equations becoming 
(from (1)),

$$
\begin{aligned}
\frac{d}{d z}(\rho v) & =0, & & \text { continuity, } \\
\frac{d}{d z}\left(\rho v^{2}+p\right) & =d_{1}, & & \text { linear momentum, } \\
\frac{d}{d z}\left(v\left(\rho e_{\text {tot }}+p\right)\right) & =d_{2}, & & \text { energy. }
\end{aligned}
$$

These three equations link the four variables $\rho(z), v(z), p(z)$, and $e_{t o t}(z)$, or combinations of them like $\mathcal{N}(z), v(z), T_{r}(z)$, and $T_{t}(z)$. The NS-system (13) cannot be solved unless completed by additional equations. A simplification widely spread in the literature consists in neglecting the dissipative terms $d_{1}$ and $d_{2}$, completing the NS-system with the equation of conservation of entropy under the assumption of isentropic flow. This approximation leads to the so-called inviscid-isentropic flow model for a perfect gas, ${ }^{30,36}$ which implies the $T_{r}=T_{t}=T$ identity of temperatures. Though useful for a coarse estimate of the thermal evolution of some jets, it is physically inconsistent since supersonic jets are essentially $T_{r} \neq T_{t}$ non-equilibrium systems. Other approaches complement the NS-system (13) with a relaxation equation (not free from inconsistencies) $)^{17,23,24,37-39}$ which relates $T_{r}$ and $T_{t}$ in terms of an effective rotational relaxation time $\tau_{r}$, cross section $\sigma_{r}$, or collision number $Z_{r}{ }^{40-42}$

The present work is based in a different philosophy. Instead of increasing the number of equations, the number of flow variables in (13) is reduced by employing those which can be determined experimentally to good accuracy, such as $\mathcal{N}(z)$ and $T_{r}(z)$. In this way the NS-system reduces to two equations in the two unknown variables, $T_{t}(z)$ and $v(z)$, providing a number of accurate relations between all four variables of the flow.

It is convenient for the present purpose to refer the NS-system (13) to molar form by means of the equivalence given in Eq. (2). Keeping this in mind, and using the equation of continuity, the equations of conservation of linear momentum, and total energy reduce to

$$
\begin{aligned}
& v \frac{d v}{d z}+\frac{1}{\mathcal{N} W} \frac{d p}{d z}=\frac{d_{1}}{\mathcal{N} W}, \\
& v \frac{d v}{d z}+\frac{1}{W} \frac{d H}{d z}=\frac{d_{2}}{v \mathcal{N} W} .
\end{aligned}
$$

Taking into account that the primary experimental data are $z, \mathcal{N}(z)$, and $T_{r}$, Eqs. (14) and (15) will be combined in order to gain as accurate as possible information about the evolution of $T_{t}(z)$ and $v(z)$ along the jet. The difference (15) minus (14) leads to the equation of gradients

$$
\frac{d H}{d z}-\frac{1}{\mathcal{N}} \frac{d p}{d z}=\mathcal{D}
$$

which provides a rigorous basis to understand the macroscopic physics in the $K n<0.3$ rarefaction regime of the jet. According to Eqs. (8)-(10), the dissipative term

$$
\mathcal{D}=\frac{d_{2}}{v \mathcal{N}}-\frac{d_{1}}{\mathcal{N}}
$$

can be expressed in terms of the transport coefficients as

$$
\mathcal{D}=\frac{1}{\mathcal{N} v}\left[\left(\frac{d v}{d z}\right)^{2}\left(\frac{4}{3} \mu+\eta_{V}\right)+\lambda \frac{d^{2} T_{t}}{d z^{2}}+\frac{d \lambda}{d T_{t}}\left(\frac{d T_{t}}{d z}\right)^{2}\right] .
$$

\section{DERIVED QUANTITIES ALONG THE JET}

In order to attain results of wider applicability a generic binary gas mixture of a single molecular species $\mathrm{M}$ and a single atomic species A will be considered, with molar fractions $\alpha$ and $1-\alpha$, 
respectively. Moreover, it will be assumed that the vibrational degrees of freedom of species $\mathrm{M}$ in the $\alpha \mathrm{M}+(1-\alpha) \mathrm{A}$ gas mixture are frozen and do not contribute with energy to the expansion. Only the interchange of energy between the translational and rotational degrees of freedom will be considered, a good approximation for stagnation temperature below $300 \mathrm{~K}$.

In that region of the jet where a high fraction (say $>99 \%$ ) of the rotational population of molecular species $\mathrm{M}$ nearly obeys a Boltzmann distribution at the temperature $T_{r}$, Eq. (16) can be written to good approximation in terms of $\mathcal{N}, T_{t}$, and $T_{r}$. Molecules such as $\mathrm{H}_{2}, \mathrm{HD}, \mathrm{D}_{2}$, and $\mathrm{CH}_{4}$, with a rotational constant $B>10 \mathrm{~cm}^{-1}$ do not satisfy this condition in supersonic jets and will be excluded from the discussion.

\section{A. Translational temperature}

Expressing the enthalpy of the gas mixture along the jet as

$$
H=\alpha\left(H_{t, \mathrm{M}}+H_{r, \mathrm{M}}\right)+(1-\alpha) H_{t, \mathrm{~A}},
$$

where $H_{t}$ and $H_{r}$ refer to the translational and rotational contributions to the enthalpy, and expressing its gradient as

$$
\frac{d H}{d z}=\alpha\left(\frac{d H_{t, \mathrm{M}}}{d T_{t}} \cdot \frac{d T_{t}}{d z}+\frac{d H_{r, \mathrm{M}}}{d T_{r}} \cdot \frac{d T_{r}}{d z}\right)+(1-\alpha)\left(\frac{d H_{t, \mathrm{~A}}}{d T_{t}} \cdot \frac{d T_{t}}{d z}\right),
$$

and taking into account that the translational (molar) heat capacity at constant pressure

$$
C_{t, p}=\frac{d H_{t, \mathrm{M}}}{d T_{t}}=\frac{d H_{t, \mathrm{~A}}}{d T_{t}}=\frac{5}{2} R
$$

is constant for all molecules and atoms, and that the rotational (molar) heat capacity is, at constant pressure and at constant volume,

$$
C_{r}=\frac{d H_{r, \mathrm{M}}}{d T_{r}}
$$

one obtains the gradient of enthalpy for the gas mixture

$$
\frac{d H}{d z}=\frac{5}{2} R \frac{d T_{t}}{d z}+\alpha C_{r} \frac{d T_{r}}{d z} .
$$

Up to not too low temperature the approximation $C_{r}=N R / 2$ holds, where $N=2$ or $N=3$ is the number of rotational degrees of freedom of linear and nonlinear molecules, respectively. At lower temperature $C_{r}$ can be calculated accurately as shown in Appendix A.

Explicit expressions for the translational temperature along the jet in terms of the experimental quantities $\mathcal{N}$ and $T_{r}$ can be derived combining Eqs. (16) and (23). This leads to the linear differential equation

$$
\frac{d T_{t}}{d z}+f(z) T_{t}=g(z)
$$

where

$$
f(z)=-\frac{2}{3 \mathcal{N}} \frac{d \mathcal{N}}{d z}
$$

and

$$
g(z)=\frac{2}{3 R}\left(\mathcal{D}-\alpha C_{r} \frac{d T_{r}}{d z}\right)
$$

Its general solution is

$$
T_{t}(z)=e^{-G(z)}\left(c+\int e^{G(z)} g(z) d z\right)
$$


with

$$
G(z)=\int f(z) d z
$$

For the boundary conditions $\mathcal{N}=\mathcal{N}_{0}$ and $T_{t}=T_{0}$ at $z=z_{0}$, one obtains

$$
G(z)=-\frac{2}{3} \ln \left(\frac{\mathcal{N}}{\mathcal{N}_{0}}\right),
$$

the translational temperature becoming eventually

$$
T_{t}(z)=\left(\frac{\mathcal{N}}{\mathcal{N}_{0}}\right)^{2 / 3}\left[T_{0}+\frac{2}{3 R} \int_{z_{0}}^{z}\left(\frac{\mathcal{N}}{\mathcal{N}_{0}}\right)^{-2 / 3}\left(\mathcal{D}-\alpha C_{r} \frac{d T_{r}}{d z}\right) d z\right],
$$

where $\mathcal{N}_{0}, T_{0}$, and $z_{0}$ are the stagnation number density, temperature, and expansion origin, respectively. Since the first data points very close to or upstream to the nozzle orifice in the integrand of (30) are difficult or even impossible to measure, the integration can run in practice from a point $z_{e}$ where $T_{r}=T_{t}$ to experimental uncertainty, replacing $\mathcal{N}_{0}$ by $\mathcal{N}\left(z_{e}\right), T_{0}$ by $T_{r}\left(z_{e}\right)$, and $z_{0}$ by $z_{e}$.

Everything in the right-hand term of Eq. (30) can be obtained from the experiment, including an estimate for $\mathcal{D}$ if the transport coefficients $\mu, \eta_{V}$, and $\lambda$ of the gas mixture are known. The estimate for $\mathcal{D}$ can be obtained from Eq. (18) employing there the inviscid-adiabatic approximation $(\mathcal{D}=0)$ for $T_{t}$ and $v$. However, a different and more systematic procedure is described in Sec. IV and Appendix B.

For a wide range of jet conditions $\mathcal{D}$ is far smaller than $\alpha C_{r} d T_{r} / d z$ and can be neglected in Eq. (30). If so, since $\mathcal{D}$ is always positive, and $d T_{r} / d z$ is always negative in the zone of silence of the jet, the translational temperature resulting from Eq. (30) for the inviscid-adiabatic approximation is slightly lower than the true translational temperature. In summary, Eq. (30), with $\mathcal{D}=0$, sets a lower boundary for the translational temperature. In the discussion below, the translational temperature derived from the inviscid-adiabatic approximation $(\mathcal{D}=0)$ will be referred to as $T_{t}^{i a}$.

\section{B. Flow velocity}

For $\mathcal{N}$ and $T_{r}$ known from the experiment, and $T_{t}$ derived from them as described above, the local flow velocity $v$ can be obtained integrating (15). However, no simple explicit expressions seem possible due to the dissipative term $d_{2}$. For $d_{2}=0$, the inviscid-adiabatic approximation leads to

$$
v^{i a}(z)=\sqrt{\frac{5 R}{W}\left(T_{0}-T_{t}^{i a}(z)\right)+\frac{2 \alpha}{W} \int_{T_{r}(z)}^{T_{0}} C_{r}(z) d T_{r}} .
$$

Since $T_{t}^{i a}$ is slightly smaller than the true translational temperature $T_{t}$, Eq. (31) slightly overestimates the true flow velocity.

Along the wide integration domain $\left[T_{0}, T_{r}\right]$ where $C_{r}(z)$ remains constant, Eq. (31) simplifies to

$$
v^{i a}(z)=\sqrt{\frac{R}{W}\left(5\left(T_{0}-T_{t}^{i a}\right)+2 \alpha \frac{C_{r}}{R}\left(T_{0}-T_{r}\right)\right)} .
$$

For not too light molecular species, with a rotational constant $B<10 \mathrm{~cm}^{-1}$, the condition $T_{r}(z)$ $\approx T_{t}^{i a}(z)$ usually holds along the first diameters downstream from the nozzle, leading to the well known approximation

$$
v^{i a}(z) \approx \sqrt{\frac{R}{W}\left(5+2 \alpha \frac{C_{r}}{R}\right)\left(T_{0}-T_{t}^{i a}\right)},
$$

which is useful for some purposes, but is not accurate far downstream the jet under severe $T_{r} \neq T_{t}$ non-equilibrium conditions. 


\section{Relationship between $\mathcal{N}$ and $T_{t}^{i a}$}

Differentiating (33) and substituting into (14) under the conditions mentioned, $T_{r} \approx T_{t}^{i a}$ and constant $C_{r}$, the relation

$$
\mathcal{N} \cdot\left(T_{t}^{i a}\right)^{-\left(\frac{3}{2}+\alpha \frac{C_{r}}{R}\right)} \approx K,
$$

between $\mathcal{N}(z)$ y $T_{t}^{i a}(z)$ is obtained for the inviscid-adiabatic approximation; $K$ is a constant. Since the heat capacity of the gas mixture at constant pressure and at constant volume is given by

$$
C_{p}=\frac{5}{2} R+\alpha C_{r} \quad \text { and } \quad C_{v}=\frac{3}{2} R+\alpha C_{r},
$$

the heat capacity ratio

$$
\gamma\left(T_{r}\right)=\frac{C_{p}}{C_{v}}=\frac{\frac{5}{2} R+\alpha C_{r}}{\frac{3}{2} R+\alpha C_{r}}
$$

remains also constant along the jet section where $C_{r}$ is constant. In this case, Eq. (34) can be expressed in terms of the stagnation conditions as

$$
\mathcal{N} \cdot\left(T_{t}^{i a}\right)^{\frac{1}{1-\gamma}} \approx \mathcal{N}_{0} \cdot T_{0}^{\frac{1}{1-\gamma}}
$$

Rewritten as

$$
T_{t}^{i a}(z) \approx T_{0}(z)\left(\frac{\mathcal{N}(z)}{\mathcal{N}_{0}(z)}\right)^{\gamma-1}
$$

it allows for an estimate of the translational temperature employing only the experimental number density $\mathcal{N}(z)$.

Equation (37) is formally similar to that of an isentropic process. It shows that the expansion of a real gas mixture through an ideal nozzle is nearly isentropic in the first section of the jet, as far the following conditions are satisfied:

- Negligible dissipative effects $\left(\mathcal{D} \ll C_{r} d T_{r} / d z\right)$;

- $T_{r}$ defined by a near-Boltzmann distribution of the rotational populations $P_{i}$;

- $T_{r} \approx T_{t}$;

- $C_{r}\left(T_{r}\right) \approx$ constant.

From the above arguments, the estimate for $T_{t}^{i a}$ of Eq. (38) is valid just for the initial section of the jet. However, as shown next, a similar equation holds in a wider range of the jet for viscous non-adiabatic and non-isentropic flow.

\section{ENTROPY ALONG THE JET}

In many works dealing with supersonic jets the isentropic flow approximation is implicitly or explicitly accepted as a good option for the paraxial region. ${ }^{23,26,30,36}$ Moreover, it is often assumed that the inviscid-adiabatic approximation is equivalent to the isentropic one. These assumptions, which may be a good approximation in some particular cases, are not true in general and may lead to systematic interpretative errors in other cases.

Since the flow variables in jets may vary by orders of magnitude depending on the stagnation conditions, nature of the expanded gas, and distance to the nozzle, it is not easy to conclude a priori about the evolution of entropy along a particular jet. Hence, a rigorous discussion of these points is pertinent, as is too the relation between entropy and translational temperature.

Let us express the (molar) entropy of the $\alpha \mathrm{M}+(1-\alpha) \mathrm{A}$ gas mixture along the jet as

$$
S(z)=C+\alpha\left(S_{t, \mathrm{M}}+S_{r, \mathrm{M}}\right)+(1-\alpha) S_{t, \mathrm{~A}},
$$

where $C$ is a constant; $S_{t, \mathrm{M}}$ and $S_{r, \mathrm{M}}$ refer to the entropy of the translational and rotational degrees of freedom of molecular species $\mathrm{M}$, and $S_{t, \mathrm{~A}}$ to the entropy of the translational degrees of freedom of atomic species A. 
Starting from the partition functions of statistical mechanics, the translational contributions to the entropy can be written in the form ${ }^{43,44}$

$$
S_{t, \mathrm{M}}=C_{t, \mathrm{M}}+R \ln \left(A_{\mathrm{M}} \frac{T_{t}^{3 / 2}}{\mathcal{N}_{\mathrm{M}}}\right)
$$

and

$$
S_{t, \mathrm{~A}}=C_{t, \mathrm{~A}}+R \ln \left(A_{\mathrm{A}} \frac{T_{t}^{3 / 2}}{\mathcal{N}_{\mathrm{A}}}\right),
$$

where $C_{t, \mathrm{M}}$ and $C_{t, \mathrm{~A}}$ are constants; $\mathcal{N}_{\mathrm{M}}$ and $\mathcal{N}_{\mathrm{A}}$ are the number densities of species $\mathrm{M}$ and $\mathrm{A}$, with

$$
A_{\mathrm{M}}=\frac{1}{N_{\mathrm{Av}}}\left(\frac{2 \pi m_{\mathrm{M}} k}{h^{2}}\right)^{3 / 2} \text { and } A_{\mathrm{A}}=\frac{1}{N_{\mathrm{Av}}}\left(\frac{2 \pi m_{\mathrm{A}} k}{h^{2}}\right)^{3 / 2},
$$

where $m_{M}$ and $m_{A}$ stand for the mass of species $\mathrm{M}$ and $\mathrm{A}$, respectively.

For $T_{r}$ high enough for the classical rotational partition function to be a good approximation, the rotational contribution to the entropy of species $\mathrm{M}$ with $N$ rotational degrees of freedom is ${ }^{45}$

$$
S_{r, \mathrm{M}}=C_{r, \mathrm{M}}+\frac{N}{2} R \ln \left(\frac{T_{r}}{\Theta_{r}}\right),
$$

where $C_{r, \mathrm{M}}$ is a constant, and $\Theta_{r}$ is the characteristic rotational temperature. For linear molecules $N=2$ and $\Theta_{r}=h^{2} / 8 \pi I_{b} k$, while for nonlinear molecules $N=3$ and $\Theta_{r}=\left(\Theta_{a} \Theta_{b} \Theta_{c}\right)^{1 / 3}$, where $\Theta_{a}$ $=h^{2} / 8 \pi I_{a} k, \Theta_{b}=h^{2} / 8 \pi I_{b} k$, and $\Theta_{c}=h^{2} / 8 \pi I_{c} k ; I_{a}, I_{b}$, and $I_{c}$ are the molecular inertia moments. Assuming no slip effects between species $\mathrm{M}$ and $\mathrm{A}$, i.e., under constant number density ratio $\mathcal{N}_{\mathrm{M}} / \mathcal{N}_{\mathrm{A}}$ along the expansion, the entropy of the mixture at a point $z$ of the jet can eventually be expressed as

$$
S(z)=C+R\left[\alpha \ln \left(\frac{A_{\mathrm{M}} T_{t}^{3 / 2}}{\mathcal{N}_{\mathrm{M}}}\right)+(1-\alpha) \ln \left(\frac{A_{\mathrm{A}} T_{t}^{3 / 2}}{\mathcal{N}_{\mathrm{A}}}\right)+\alpha \frac{N}{2} \ln \left(\frac{T_{r}}{\Theta_{r}}\right)\right],
$$

while

$$
S_{0}=C+R\left[\alpha \ln \left(\frac{A_{\mathrm{M}} T_{0}^{3 / 2}}{\mathcal{N}_{\mathrm{M}, 0}}\right)+(1-\alpha) \ln \left(\frac{A_{\mathrm{A}} T_{0}^{3 / 2}}{\mathcal{N}_{\mathrm{A}, 0}}\right)+\alpha \frac{N}{2} \ln \left(\frac{T_{0}}{\Theta_{r}}\right)\right]
$$

is the stagnation entropy under the stagnation conditions $\mathcal{N}_{0}$ and $T_{r 0}=T_{t 0}=T_{0}$. The increment of entropy along the jet

$$
\Delta S=S(z)-S_{0}
$$

follows from Eqs. (44) and (45). Taking into account that $\mathcal{N}_{\mathrm{M}} / \mathcal{N}_{\mathrm{M}, 0}=\mathcal{N}_{\mathrm{A}} / \mathcal{N}_{\mathrm{A}, 0}=\mathcal{N} / \mathcal{N}_{0}=$, this leads to the general expression

$$
\frac{\Delta S}{R}=\ln \left(\frac{\mathcal{N}_{0}}{\mathcal{N}} \frac{T_{t}^{\frac{3}{2}+\alpha \frac{N}{2}}}{T_{0}^{\frac{3}{2}+\alpha \frac{N}{2}}} \frac{T_{r}^{\alpha \frac{N}{2}}}{T_{t}^{\alpha \frac{N}{2}}}\right),
$$

where all particular atomic and molecular properties, such as $m_{\mathrm{A}}, m_{\mathrm{M}}, I_{a}, I_{b}, I_{c}$, have cancelled out. On the other hand, taking into account that

$$
\frac{3}{2}+\alpha \frac{N}{2}=\frac{1}{\gamma-1}
$$

Eq. (47) can be inverted in order to express the local translational temperature as

$$
T_{t}(z)=T_{0}\left(\frac{\mathcal{N}}{\mathcal{N}_{0}}\right)^{\gamma-1} F
$$

where

$$
F=\frac{e^{(\gamma-1) \frac{\Delta S}{R}}}{\left(T_{r} / T_{t}\right)^{\frac{5-3 \gamma}{2}}}
$$


is a functional form suitable for iterative numerical calculation as shown in Appendix B. For $F=$ 1, Eq. (50) corresponds to Eq. (38). The value $F=1$ has, however, two solutions. One solution is the trivial one, with $\Delta S=0$ and $T_{r}=T_{t}$, which corresponds to the isentropic flow with negligible breakdown of rotation-translation equilibrium proper of the very beginning of the expansion, as discussed in Sec. III C. The other $F=1$ solution is obtained for the relation between entropy increment $\Delta S$ and $T_{r} \neq T_{t}$ non-equilibrium given by the condition

$$
\frac{\Delta S}{R}=\ln \left(\frac{T_{r}}{T_{t}}\right)^{\alpha \frac{N}{2}}
$$

which may occur accidentally, with the result that the translational temperature in highly nonisentropic jets with severe $T_{r} \neq T_{t}$ non-equilibrium might be very close to the isentropic or the inviscid-adiabatic temperature $T_{t}^{i a}$ of Eq. (38). Actually, since $\Delta S$ and $T_{r} / T_{t}$ are correlated (see below), this behavior may occur occasionally.

In the general case, the numerator (num) and denominator (den) in Eq. (50) start at the value $n u m=d e n=1$ at the beginning of the expansion and are always positive, growing correlated along the expansion, with no maxima nor minima, with the result that function $F$ tends to remain $F \approx 1$ along the expansion. This explains the paradox that the translational temperature of non-isentropic jets with $T_{r} \neq T_{t}$ non-equilibrium sometimes tends to behave as if they were isentropic. ${ }^{7}$ As we shall see next, all jets are to some extent non-isentropic.

An insight into the origin of entropy along the jet is possible deriving (44), with the result

$$
\frac{d S}{d z}=R\left(\frac{3}{2} \frac{1}{T_{t}} \frac{d T_{t}}{d z}-\frac{1}{\mathcal{N}} \frac{d \mathcal{N}}{d z}\right)+\alpha \frac{N}{2} \frac{R}{T_{r}} \frac{d T_{r}}{d z},
$$

where the equivalence

$$
\left(\frac{3}{2} \frac{1}{T_{t}} \frac{d T_{t}}{d z}-\frac{1}{\mathcal{N}} \frac{d \mathcal{N}}{d z}\right)=\frac{\mathcal{D}}{R T_{t}}-\alpha \frac{N}{2} \frac{1}{T_{t}} \frac{d T_{r}}{d z}
$$

obtained from Eq. (16) will be used for simplification. Substituting the left-hand term into (52), one obtains eventually

$$
\frac{d S}{d z}=\frac{\mathcal{D}}{T_{t}}-\alpha C_{r}\left(\frac{1}{T_{t}}-\frac{1}{T_{r}}\right) \frac{d T_{r}}{d z},
$$

which shows explicitly the two sources of entropy along the jet, one arising from the dissipation $\mathcal{D}$, and the other from the $T_{r} \neq T_{t}$ non-equilibrium. Both contributions are non-negative, the first one because $\mathcal{D}>0$, as results from Eq. (18) for all atoms and molecules, and the second because $T_{r}>T_{t}$, and $d T_{r} / d z \leq 0$ along the zone of silence of any jet. Note that even under the inviscidadiabatic approximation $(\mathcal{D}=0)$, the jet is non-isentropic as far as $d T_{r} / d z \neq 0$, since the condition $T_{r}>T_{t}$ always holds, with increasing difference $T_{r}-T_{t}$ downstream along the jet. When $T_{r}$ tends to freeze $\left(d T_{r} / d z \rightarrow 0\right)$, the flow tends to behave as monoatomic. Moreover, since $\mathcal{D} / T_{t}$ decreases fast downstream along the jet, the far downstream flow tends to become isentropic.

The relative importance of the $T_{r} \neq T_{t}$ non-equilibrium contribution to entropy in Eq. (54), compared to dissipation contribution $\mathcal{D}$, very much depends on the particular jet considered. First, the $T_{r} \neq T_{t}$ contribution depends linearly on the molar fraction $(\alpha)$ of molecules in the jet. Thus, this contribution may be expected to be more important in pure molecular jets than in molecular jets highly diluted in atomic species. Second, the $T_{r} \neq T_{t}$ contribution depends on the $T_{r}-T_{t}$ difference, which is governed by the inelastic collision properties of the molecules in the jet. ${ }^{3-8}$ As a general rule, light molecules such as $\mathrm{H}_{2}$ show much larger $T_{r}-T_{t}$ differences than heavier molecules such as $\mathrm{N}_{2}, \mathrm{O}_{2}$, or $\mathrm{H}_{2} \mathrm{O}$. On the other hand, the $d T_{r} / d z$ gradient in (54) is also regulated by inelastic collisions, and is large in the initial section, and non-negligible in the medium section of the jets.

In the whole, it can be expected that the $T_{r} \neq T_{t}$ contribution to the evolution of entropy along the jets may be important, specially in jets with high proportion of the relevant species $\mathrm{H}_{2}, \mathrm{~N}_{2}, \mathrm{O}_{2}$, and $\mathrm{H}_{2} \mathrm{O}$. This suggests that the $T_{r} \neq T_{t}$ non-equilibrium is irreversible and appears thus spontaneously in all molecular jets with no exception. 
It must be emphasized that both contributions to the increment of entropy according to Eq. (54), dissipation $\mathcal{D}$ and $T_{r} \neq T_{t}$ non-equilibrium, depend on the same microscopic events, the inelastic collisions. According to Eq. (18), the dissipation $\mathcal{D}$ depends on shear $(\mu)$ and bulk $\left(\eta_{V}\right)$ viscosities, and on thermal conductivity $(\lambda)$. The contribution of inelastic collisions to $\mu$ may amount up to $15 \%,{ }^{42} \eta_{V}$ depends entirely on inelastic collisions, ${ }^{52,53}$ and $\lambda$ depends also to a good extent on them. ${ }^{41}$ Eventually, it may be concluded that the ultimate reason for irreversibility and $T_{r} \neq T_{t}$ non-equilibrium in supersonic jets are the microscopic inelastic collision events they depend on.

However, Eq. (54) is only accurate for $T_{r}$ high enough, i.e., as far as the classical rotational partition function remains a good approximation. For low $T_{r}$ s the exact definition of rotational entropy

$$
S_{r, \mathrm{M}}=S_{r}^{0}+R \sum_{J} P_{J} \ln \left(\frac{\omega_{J}(2 J+1)}{P_{J}}\right)
$$

in terms of the populations $P_{J}$ of the rotational quantum levels should be employed; $J$ is the angular momentum quantum number, and $\omega_{J}$ is the statistical weight of the rotational level. Somewhat more involved expressions in terms of rotational populations instead of rotational temperature are obtained in this case.

Equation (54) shows that under the inviscid-adiabatic approximation $(\mathcal{D}=0)$, the jet starts and remains isentropic as far $T_{r} \approx T_{t}$, in agreement with the conditions expressed in Sec. III C. However, real jets with non-negligible dissipation $\mathcal{D}>0$ are non-isentropic from the very beginning of the expansion, then entropy increases due to the $T_{r} \neq T_{t}$ non-equilibrium, and eventually tend to behave isentropic if the $\mathcal{D} / T_{t} \rightarrow 0$ and $d T_{r} / d z \rightarrow 0$ asymptotic conditions hold.

Whether the increment $\Delta S$ is negligible or large along the jet markedly depends on the size of the nozzle orifice and distance to it, on the nature of the expanded gas, and on the stagnation conditions, as illustrated in Sec. V.

\section{A REFERENCE CASE: DISSIPATION IN PURE HELIUM JETS}

Since helium is often employed as carrier gas in highly diluted $\alpha \mathbf{M}+(1-\alpha) \mathrm{He}$ laboratory jets for efficient cooling purpose and, furthermore, such jets provide the natural environment for the study of M:He inelastic collisions, ${ }^{8}$ it is worth exploring the behavior of translational temperature and entropy in pure helium jets according to the above guidelines using the procedure outlined in Appendix B.

The thermal and entropic behavior of pure helium free jets expanded through a circular orifice of diameter $D=350 \mu \mathrm{m}$ at stagnation temperature $T_{0}=360 \mathrm{~K}$ and stagnation pressures between $p_{0}=10 \mathrm{mbars}$ and $p_{0}=100 \mathrm{mbars}$ is explored. These conditions correspond to recent experiments aimed at the investigation of $\mathrm{H}_{2} \mathrm{O}: \mathrm{He}$ inelastic collisions. ${ }^{8}$

Since the reduced number density $\mathcal{N} / \mathcal{N}_{0}$ along the jets is very nearly independent on stagnation conditions, the values resulting from the Mach number parameterization ${ }^{30}$ for $\gamma=5 / 3$ will be employed here as a starting point. As follows from Eq. (B1) of Appendix B, the zero order approximation of the reduced translational temperature, $T_{t}^{(0)} / T_{0}$, which is equal to the reduced isentropic temperature, is also independent on stagnation conditions. Both quantities are shown in Fig. 1. The first order approximation of dissipation $\mathcal{D}$, entropy increment $\Delta S$, and translational temperature $T_{t}$ are shown in Figs. 2-4, respectively.

The 1st order dissipation $\mathcal{D}^{(1)}$ shown in Fig. 2 has been calculated from Eq. (B3) of Appendix B employing the zero order translational temperature and velocity, $T_{t}^{(0)}$ and $v^{(0)}$, and the shear viscosity $\mu\left(T_{t}^{(0)}\right)$ and thermal conductivity $\lambda\left(T_{t}^{(0)}\right)$ of helium, ${ }^{46}$ while the bulk viscosity $\eta_{V}=0$ has been assumed. The contribution of viscosity to the dissipation of helium jets shown in Fig. 2 is important just at the very beginning of the expansion and decays very fast with distance to the nozzle; at $z / D=0$ viscosity and thermal conductivity contributions amount about the same, however at $z / D=1$ the shear viscosity contributes with only $10 \%$ to dissipation, and at $z / D=2$ with $3 \%$. For $z / D>3$ just the high thermal conductivity of helium matters. 


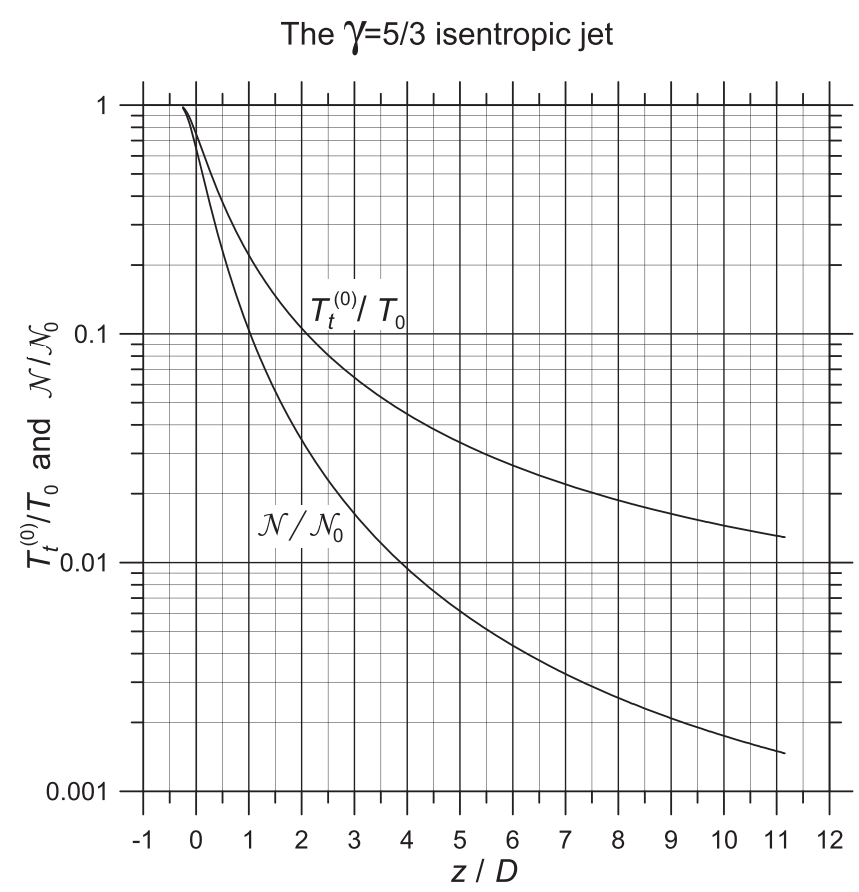

FIG. 1. Reduced number density and zeroth order (isentropic) translational temperature along He supersonic free jets.

The 1st order contribution to the increment of entropy $\Delta S^{(1)}$ shown in Fig. 3 has been calculated from Eq. (B4) of Appendix B, where the term in $\alpha C_{r}$ vanishes for pure atomic jets. As shown in Fig. 2, the dissipation $\mathcal{D}^{(1)}$ is inversely proportional to the stagnation pressure and decreases fast along the expansions. However, the integral of $\mathcal{D} / T_{t}$ in Eqs. (54) and (B4) keeps growing due to the decrease of $T_{t}$ along the expansion, leading to the smooth increment of entropy shown in Fig. 3,

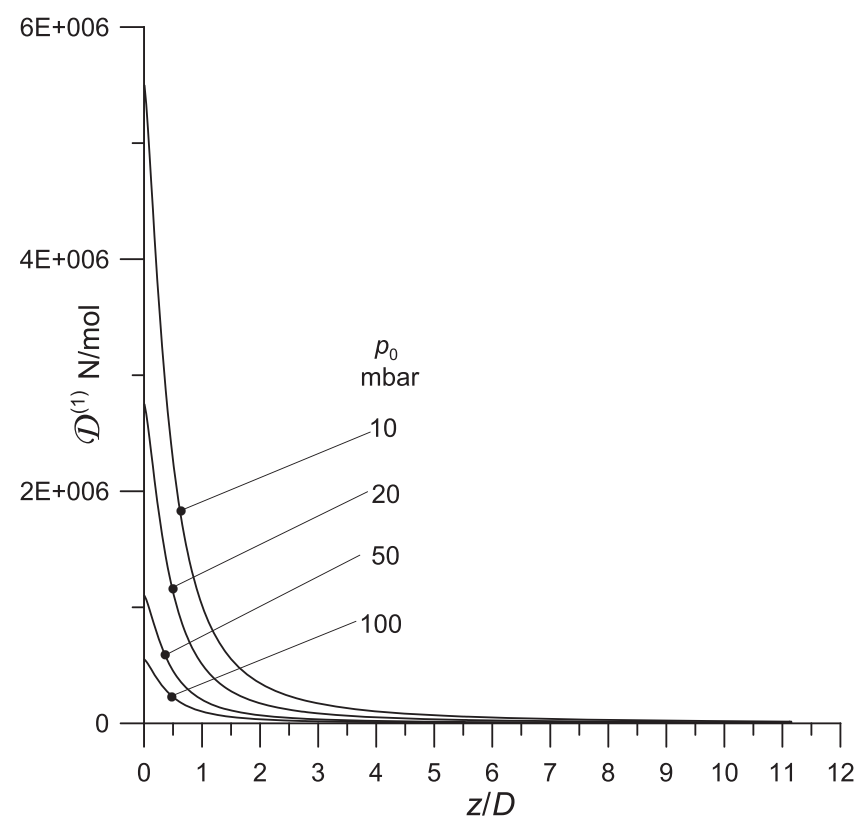

FIG. 2. Dissipation $\mathcal{D}^{(1)}$ (to first order) in He supersonic free jets expanded through an ideal $D=350 \mu \mathrm{m}$ orifice at stagnation temperature $T_{0}=360 \mathrm{~K}$ and pressures $p_{0}$. 


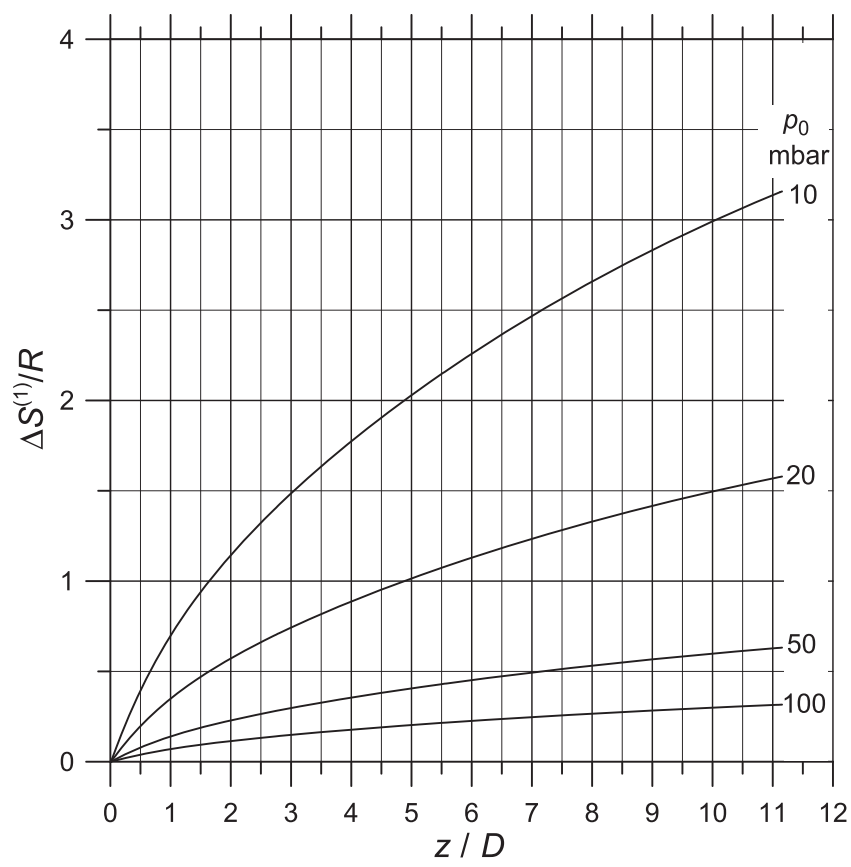

FIG. 3. Increment of entropy (to first order) in He supersonic free jets expanded through an ideal $D=350 \mu \mathrm{m}$ orifice at stagnation temperature $T_{0}=360 \mathrm{~K}$ and pressures $p_{0}$.

which is also inversely correlated with the stagnation pressure. Helium free jets expanded under $p_{0}$ $>200$ mbar through a $D=350 \mu \mathrm{m}$ orifice are nearly isentropic, while $p_{0}<100$ mbar jets clearly depart from the isentropic behavior, the more the lower the stagnation pressure.

The 1st order translational temperatures $T_{t}^{(1)}$ shown in Fig. 4 have been calculated by means of function $F^{(1)}$ employing Eqs. (B7) and (B5) of Appendix B. The translational temperature of helium

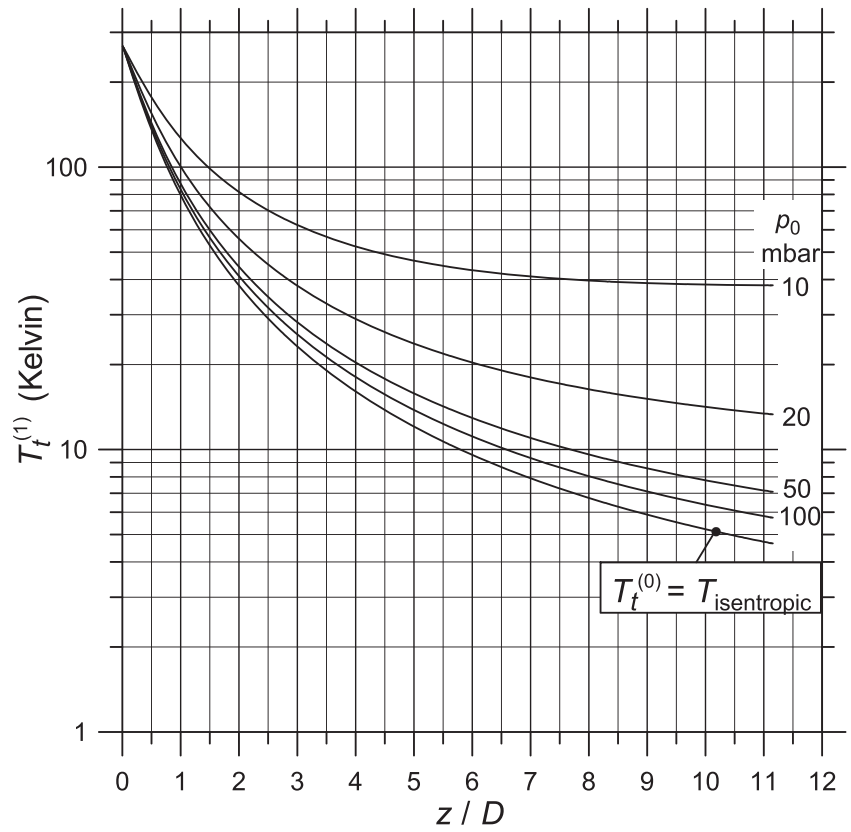

FIG. 4. Zero order translational temperature $\left(T_{t}^{(0)}=T_{\text {isentropic }}\right)$ and first order translational temperature $\left(T_{t}^{(1)}\right)$ in He supersonic free jets expanded through an ideal $D=350 \mu \mathrm{m}$ orifice at stagnation temperature $T_{0}=360 \mathrm{~K}$ and pressures $p_{0}$. 
jets expanded under $p_{0}>200$ mbar differ from the isentropic temperature by less than $1 \mathrm{~K}$, but for $p_{0}<100 \mathrm{mbar}$ the departure from the isentropic temperature can be strong due to the exponential effect of the entropy increment on factor $F$, according to Eqs. (50) and (B5). A qualitatively similar increment of $T_{t}$ with respect to the isentropic flow has been reported in a study of terminal speed ratios in highly expanded helium jets. ${ }^{27}$ The quantum effects described in the far downstream region of those jets ${ }^{27}$ are, however, beyond the range of validity of the present equations due to the large local Knudsen number. As far as a comparison with neon jets highly diluted in helium can be meaningful, a similar incremental trend for terminal $T_{t} \mathrm{~s}$ as a function of stagnation pressure has been reported. ${ }^{47}$

The convergence of the iterative procedure described in Appendix B also depends on stagnation pressure $p_{0}$, with enhanced convergence the higher $p_{0}$.

It must be stressed that the results shown in Figs. $2-4$ at a particular fixed position $z / D$ of the jets depend on the orifice diameter $D$, in contrast with Fig. 1, which is invariant with respect to $D$. This can be deduced from Eq. (16). If expressed in terms of the reduced distances $\tilde{z}=z / D$, the equation of gradients (16) shows a left-hand term independent on $D$ and a right-hand dissipation $\mathcal{D}(\tilde{z})$ term inversely proportional to $D$. Hence, at the same $z / D$ position, jets expanded through smaller orifices are more prone to dissipative effects, showing larger increments of entropy and larger departure of the translational temperature from the isentropic one. Jets through larger $D$ s, on the contrary, tend to behave more isentropic. A comparable behavior can be expected for jets of other noble gases. Although their heat conductivities are smaller than for helium, the larger molecular mass reduces subquadratically the flow velocity, globally increasing the dissipation according to Eqs. (18) and (B3).

\section{COMMENTS ON THE PERFECT GAS MODEL FOR SUPERSONIC JETS}

The perfect gas model (or isentropic model) of a supersonic jet is widely referred to in the literature, ${ }^{25,30,36}$ however, with little or no assessment at all of its merits and limitations. Comments on this model are pertinent here. The perfect gas hypothesis implies the identity of rotational and translational temperatures. Imposing the perfect gas conditions $\left(\mathcal{D}=0\right.$ and $\left.T_{r}=T_{t}=T\right)$ in Eq. (16), jointly with the definition of Mach number $M=v / a$, the model leads to the well-known relations

$$
\mathcal{N}=\mathcal{N}_{0}\left(1+M^{2}(\gamma-1) / 2\right)^{\frac{1}{1-\gamma}}
$$

and

$$
T=T_{0}\left(1+M^{2}(\gamma-1)\right)^{-1},
$$

which satisfy the isentropic relation (37) between $\mathcal{N}$ and $T$. Solving the NS-equations by the method of characteristics for the perfect gas conditions, the Mach number can be expressed as a function ${ }^{25,30}$

$$
M(\tilde{z})=\tilde{z}^{\gamma-1}\left(b+c / \tilde{z}+d / \tilde{z}^{2}+e / \tilde{z}^{3}\right)
$$

of the reduced distance $\tilde{z}=z / D$, and parameters $b, c, d, e$ which depend on $\gamma \cdot{ }^{10,30}$

As it has been shown in Sec. III C, the above conditions just hold under highly efficient rot-trans transfer of energy proper of the initial section of some jets, i.e., under very slight $T_{r} \neq T_{t}$ nonequilibrium and negligible dissipative effects. Abundant experimental material on $\mathrm{H}_{2}, \mathrm{H}_{2}+\mathrm{He}, \mathrm{N}_{2}$, $\mathrm{O}_{2}, \mathrm{CO}_{2}, \mathrm{CO}, \mathrm{N}_{2}+\mathrm{H}_{2}$ jets ${ }^{2-7,24,48}$ strongly suggests that this condition is satisfied just by species of molecular mass above $25 \mathrm{u}^{49}$ at not too low stagnation pressures. The prototype of this behavior are $\mathrm{N}_{2}$ jets at stagnation pressures in the range $0.2<p_{0}<3$ bar and stagnation temperatures $T_{0}$ $\approx 300 \mathrm{~K}$ expanded through a sub-millimeter orifice. Their number densities $\mathcal{N}$ are well accounted for by (56) and (58) with $\gamma=7 / 5$, but the description of the thermal behavior along the jets by (57) is uncertain as the perfect gas model does not consider at all the $T_{r} \neq T_{t}$ non-equilibrium. Above moderately high stagnation pressures, Eq. (57) may provide a good approximation for the translational temperature $T_{t}$, but fails in describing the rotational temperature $T_{r}$. For light molecular species or mixtures $(<25 \mathrm{u})$, Eqs. (56) and (57) fail even at short distances $(z / D<1)$ from the nozzle. An additional limitation of the perfect gas model concerns the nozzle diameter $D$ implicit in 
Eq. (58), which is often difficult to establish with accuracy in real free jets due to the boundary layer effects at the orifice. Indeed, real free jets may strongly depart from isentropic behavior as has been shown in Secs. IV and V, depending on source conditions, distance to nozzle orifice, and expanded gas.

The perfect gas model provides, however, a good approximation for $T_{t}^{(0)}$ and $v^{(0)}$, which are required jointly with $\mathcal{D}^{(0)}=0, \Delta S^{(0)}=0$, and $F^{(0)}=1$ conditions for the iterative calculation of the translational temperature, flow velocity, dissipation, and increment of entropy according to Appendix B.

\section{SUMMARY AND CONCLUSIONS}

Since the number density $\mathcal{N}$ and rotational temperature $T_{r}$ are amenable to accurate experimental determination along supersonic free jets, emphasis has been made here onto the translational temperature $T_{t}$ and entropy increment $\Delta S$, which cannot be determined directly from experiment. The influence of dissipative effects onto $T_{t}$ and $\Delta S$ has been analyzed in detail on the basis of NS-equations. Approximations are discussed, and closed equations and procedures are proposed for the determination of $T_{t}$ and $\Delta S$ along the jets on the basis of $\mathcal{N}$ and $T_{r}$ observables. In the equations given no parameters are employed, just quantities with unambiguous physical meaning.

The major conclusions from this work are:

(1) Two sources of entropy increment $\Delta S$ along the jets have been identified, one due to dissipation $\mathcal{D}$, and the other from the $T_{r} \neq T_{t}$ non-equilibrium in jets containing molecular species. A quantitative description of $\Delta S$ is provided by Eqs. (54) and (B4).

(2) The inviscid-adiabatic $(\mathcal{D}=0)$ and the isentropic $(\Delta S=0)$ approximations are equivalent only in supersonic jets of atomic species as proved by Eq. (54). In this case, since $\alpha=0$ in Eq. (54), $\mathcal{D}=0$ implies $\Delta S=0$.

(3) The inviscid-adiabatic approximation $\left(\mathcal{D}=0\right.$, with $\left.T_{r} \neq T_{t}\right)$ in supersonic jets containing molecular species is not isentropic, as proved by Eq. (54) where $\alpha>0$ and $d T_{r} / d z<0$ in this case.

(4) As a general rule, dissipative effects accounted for by $\mathcal{D}$ depend (through $v$ ) on the average molar mass of the expanded gas, and are inversely proportional to the stagnation pressure of the jet, $p_{0}$, and to the orifice diameter $D$. Equations (18) and (B3) account for these contributions.

(5) Accurate evaluation of dissipative effects rests upon low temperature data of the transport properties of the expanded gas (shear- and bulk viscosities, and thermal conductivity) according to Eqs. (18) and (B3).

(6) A complete and accurate characterization of the paraxial flow properties along the zone of silence of supersonic free jets is proposed combining experimental data $\left(\mathcal{N}\right.$ and $\left.T_{r}\right)$ with relations and procedures described in this work for the remaining flow quantities ( $\left.\mathcal{D}, T_{t}, v, \Delta S\right)$; Eqs. (18) and (B3) for $\mathcal{D}$; Eqs. (30), (49), (B6), and (B7) for $T_{t}$; Eqs. (31)-(33), and (B2) for $v$; Eqs. (47), (54), and (B4) for $\Delta S$.

The expressions given in the present work are aimed at improving the accuracy of experimental diagnostics of jets. Such background represents a necessary step towards the experimental study of elementary collision processes. Much experimental, theoretical, and interpretative work is still needed before this goal can be attained in a general form. In particular, the range of applicability of the present results to highly rarefied jets, far downstream in the expansion, needs to be established with precision with aid of numerical calculations and experiments. A natural extension of the present work, already in progress, suggests two tasks, (i) the microscopic formulation of temperatures and entropy along the jets in terms of elementary collision processes, and (ii) the revision of the widely employed relaxation equation, ${ }^{17,23,24,28,38,39}$ and concepts related to it like collision number, macroscopic rotational cross section, and transport coefficients under strong $T_{r} \neq T_{t}$ non-equilibrium conditions. In this respect, a manageable and rigorous formulation of these concepts in connection with supersonic jet experiments is still lacking, in spite of the wealth of ideas, methods, and formulations developed before by other authors. ${ }^{28,40,42,47,50-54}$ 


\section{ACKNOWLEDGMENTS}

This work has been supported by the Spanish Ministerio de Ciencia e Innovación (MICINN), Research Project Nos. CONSOLIDER ASTROMOL CSD2009-0038 and FIS2010-22064. Thanks are due to J. M. Fernández and G. Tejeda for so many useful comments to the paper.

\section{APPENDIX A: THE ROTATIONAL HEAT CAPACITY}

In that region of a jet where a high fraction (say $>99 \%$ ) of the rotational population nearly obeys a Boltzmann distribution at the temperature $T_{r}$, the rotational heat capacity can be calculated to very good approximation as ${ }^{42}$

$$
C_{r}=R\left(\left\langle\mathcal{E}^{2}\right\rangle-\langle\mathcal{E}\rangle^{2}\right),
$$

where

$$
\left\langle\mathcal{E}^{n}\right\rangle=Q_{r}^{-1} \sum_{i} \mathcal{E}_{i}^{n} \exp \left(-\mathcal{E}_{i}\right), \quad(n=1,2)
$$

with the rotational partition function

$$
Q_{r}=\sum_{i} \exp \left(-\mathcal{E}_{i}\right)
$$

Here $\mathcal{E}_{i}=E_{i} /\left(k_{B} T_{r}\right)$ is the reduced energy (dimensionless) of rotational state $i$, with energy $E_{i}$ referred to that of the lowest accessible rotational state. In order to avoid ambiguity with the lowest rotational energy level, ortho and para variants of molecules, such as $\mathrm{H}_{2}, \mathrm{~N}_{2}$, or $\mathrm{H}_{2} \mathrm{O}$, must be considered different species if $T_{r}$ is low enough.

\section{APPENDIX B: ITERATIVE CALCULATION OF TRANSLATIONAL TEMPERATURE, VELOCITY, DISSIPATION, AND ENTROPY}

Let us take the zero order values of dissipation $\mathcal{D}$, entropy increment $\Delta S$, and function $F$ as $\mathcal{D}^{(0)}=0, \Delta S^{(0)}=0$, and $F^{(0)}=1$, and the loop for $i=0,1,2,3 \ldots$, with

$$
T_{t}^{(i)}=T_{0}\left(\frac{\mathcal{N}}{\mathcal{N}_{0}}\right)^{\gamma-1} F^{(i)},
$$

which leads to

$$
v^{(i)}=\sqrt{\frac{R}{W}\left(5\left(T_{0}-T_{t}^{(i)}\right)+2 \alpha \frac{C_{r}}{R}\left(T_{0}-T_{r}\right)\right)}
$$

and to

$$
\begin{gathered}
\mathcal{D}^{(i+1)}=\frac{1}{\mathcal{N} v^{(i)}}\left[\left(\frac{d v^{(i)}}{d z}\right)^{2}\left(\frac{4}{3} \mu+\eta_{V}\right)+\lambda \frac{d^{2} T_{t}^{(i)}}{d z^{2}}+\frac{d \lambda}{d T_{t}^{(i)}}\left(\frac{d T_{t}^{(i)}}{d z}\right)^{2}\right], \\
\Delta S^{(i+1)}=\int_{z_{0}}^{z} \frac{\mathcal{D}^{(i+1)}}{T_{t}^{(i)}} d z-\alpha C_{r} \int_{z_{0}}^{z}\left(\frac{1}{T_{t}^{(i)}}-\frac{1}{T_{r}}\right) \frac{d T_{r}}{d z} d z, \\
F^{(i+1)}=\frac{e^{(\gamma-1) \frac{\Delta \Delta^{(i+1)}}{R}}}{\left(T_{r} / T_{t}^{(i)}\right)^{\frac{5-3 \gamma}{2}}},
\end{gathered}
$$

becoming the iterated translational temperature

$$
T_{t}^{(i+1)}=T_{0}\left(\frac{\mathcal{N}}{\mathcal{N}_{0}}\right)^{\gamma-1} F^{(i+1)}
$$


or

$$
T_{t}^{(i+1)}=T_{t}^{(0)} F^{(i+1)}
$$

where $T_{t}^{(0)}$ is the temperature of the ideal isentropic jet.

${ }^{1}$ A. Ramos, G. Tejeda, J. M. Fernández, and S. Montero, "Rotational-translational state-to-state collision rate constants of $\mathrm{N}_{2}$ at low temperature $(3<T<16 \mathrm{~K})$," Phys. Rev. A 66, 022702 (2002)

${ }^{2}$ B. Maté, F. Thibault, A. Ramos, G. Tejeda, J. M. Fernández, and S. Montero, "Experimental and theoretical determination of rotational-translational state-to-state rate constants for $\mathrm{N}_{2}$ :He collisions at low temperature $(3<T<20 \mathrm{~K})$," J. Chem. Phys. 118, 4477 (2003).

${ }^{3}$ B. Maté, F. Thibault, G. Tejeda, J. M. Fernández, and S. Montero, "Inelastic collisions in para- $\mathrm{H}_{2}$ : Translation-rotation state-to-state rate coefficients and cross sections at low temperature and energy," J. Chem. Phys. 122, 064313 (2005).

${ }^{4}$ S. Montero, F. Thibault, G. Tejeda, and J. M. Fernández, "Rotational state-to-state rates and spectral representation of inelastic collisions in low-temperature molecular hydrogen," J. Chem. Phys. 125, 124301 (2006).

${ }^{5}$ J. P. Fonfría, A. Ramos, F. Thibault, J. M. Fernández, and S. Montero, "Inelastic collisions in molecular nitrogen at low temperature $(2 \leq T \leq 50 \mathrm{~K})$," J. Chem. Phys. 127, 134305 (2007)

${ }^{6}$ G. Tejeda, F. Thibault, J. M. Fernández, and S. Montero, "Low temperature inelastic collisions between hydrogen molecules and helium atoms," J. Chem. Phys. 128, 224308 (2008).

${ }^{7}$ J. Pérez-Ríos, G. Tejeda, J. M. Fernández, M. I. Hernández, and S. Montero, "Inelastic collisions in molecular oxygen at low temperature $(4 \leq T \leq 34 \mathrm{~K})$. Close coupling calculations versus experiment," J. Chem. Phys. 134, 174307 (2011).

${ }^{8}$ G. Tejeda, J. M. Fernández, and S. Montero, "Diagnostics of $\mathrm{H}_{2} \mathrm{O}$ and $\mathrm{H}_{2} \mathrm{O}+\mathrm{He}$ supersonic jets by Raman spectroscopy," Proceedings of the 28th International Symposium on Rarefied Gas Dynamics, AIP Conference Proceedings, Vol. 1501, pp. 1305-1313 (2012), edited by M. Mareschal and A. Santos.

${ }^{9}$ G. Luijks, S. Stolte, and J. Reuss, "Molecular beam diagnostics by Raman spectroscopy,” Chem. Phys. 62, 217 (1981).

${ }^{10}$ G. Tejeda, B. Maté, J. M. Fernández-Sánchez, and S. Montero, "Temperature and density mapping of supersonic jet expansions using linear Raman spectroscopy,” Phys. Rev. Lett. 76, 34 (1996).

${ }^{11}$ B. Maté, G. Tejeda, and S. Montero, "Raman spectroscopy of supersonic jets of $\mathrm{CO}_{2}$ : Density, condensation, and translational, rotational, and vibrational temperatures,” J. Chem. Phys. 108, 2676 (1998).

${ }^{12}$ A. Ramos, B. Maté, G. Tejeda, J. M. Fernández, and S. Montero, "Raman spectroscopy of hypersonic shock waves,” Phys. Rev. E 62, 4940 (2000).

${ }^{13}$ S. Montero, B. Maté, G. Tejeda, J. M. Fernández, and A. Ramos, "Raman studies of free jet expansion (diagnostics and mapping)," in Atomic and Molecular Beams. The State of the Art 2000, edited by R. Campargue (Springer, Berlin, 2001), p. 295.

${ }^{14}$ B. Maté, I. A. Graur, T. Elizarova, I. Chirokov, G. Tejeda, J. M. Fernández, and S. Montero, "Experimental and numerical investigation of an axisymmetric supersonic jet," J. Fluid Mech. 426, 177 (2001).

${ }^{15}$ S. Montero, "Raman spectroscopic experiments on Boltzmann collision integral in supersonic jets," Proceedings of the 26th International Symposium on Rarified Gas Dynamics, AIP Conference Proceedings, Vol. 1084, pp. 3-11 (2008).

${ }^{16}$ P. V. Marrone, "Temperature and density measurements in free jets and shock waves," Phys. Fluids 10, 521 (1967).

${ }^{17}$ A. E. Belikov, R. G. Sharafutdinov, and A. V. Storozhev, "Rotational relaxation of nitrogen in helium," Chem. Phys. 213, 319 (1996).

${ }^{18}$ H. Mori, T. Niimi, I. Akiyama, and T. Tsuzuki, "Experimental detection of rotational and non-Boltzmann distribution in supersonic free molecular micro flows," Phys. Fluids 17, 117103 (2005).

${ }^{19}$ E. P. Muntz, "Measurements of anisotropic velocity distribution function in rapid radial expansions," Rarefied Gas Dynamics, Vol. 1, Proceedings of the Fifth International Symposium held at the University of Oxford, 1966, C. L. Brundin, editor (Academic Press, New York, 1967), p. 1257.

${ }^{20}$ K. A. Bütefisch and D. Vennemann, "The electron-beam technique in hypersonic rarefied gas dynamics," Prog. Aerosp. Sci. 15, 217 (1974).

${ }^{21}$ J. N. Forkey, N. D. Finkelstein, W. R. Lempert, and R. B. Miles, "Demonstration and characterization of filtered Rayleigh scattering for planar velocity measurements," AIAA J. 34, 442 (1996).

${ }^{22}$ R. B. Miles, W. R. Lempert, and J. N. Forkey, "Laser Rayleigh scattering," Meas. Sci. Technol. 12, R33 (2001).

${ }^{23}$ R. J. Gallagher and J. B. Fenn, "Relaxation rates from the time of flight analysis of molecular beams," J. Chem. Phys. 60, 3487 (1974).

${ }^{24}$ F. J. Aoiz, T. Díez-Rojo, V. J. Herrero, B. Martínez-Haya, M. Menéndez, P. Quintana, L. Ramonat, I. Tanarro, and E. Verdasco, "Low-temperature rotational relaxation of $\mathrm{N}_{2}$ studied with resonance-enhanced multiphoton ionization," J. Phys. Chem. A 103, 823 (1999).

${ }^{25}$ H. Ashkenas and F. S. Sherman, "The structure and utilization of supersonic free jets in low density wind tunnels," AIP Conf. Proc. 7, 84 (1966).

${ }^{26}$ J. B. Anderson and J. B. Fenn, "Velocity distributions in molecular beams from nozzle sources," Phys. Fluids 8, 780 (1965).

${ }^{27}$ J. P. Toennies and K. Winkelmann, "Theoretical studies of highly expanded free jets: Influence of quantum effects and a realistic intermolecular potential,” J. Chem. Phys. 66, 3965 (1977).

${ }^{28}$ L. K. Randeniya and M. A. Smith, "A study of molecular supersonic flow using the generalized Boltzmann equation," J. Chem. Phys. 93, 661 (1990).

${ }^{29}$ H. Schlichting and K. Gersten, Boundary Layer Theory, 8th ed. (Springer, Berlin, 2000).

${ }^{30}$ D. R. Miller, "Free jet sources," in Atomic and Molecular Beams, edited by G. Scoles (Oxford University Press, New York, 1988), Vol. I, p. 14 
${ }^{31}$ G. Millán, Problemas Matemáticos de la Mecánica de Fluidos (Real Acad. Ciencias Exactas, Físicas y Naturales, Madrid, 1975).

${ }^{32}$ T. G. Elizarova, A. A. Khokhlov, and S. Montero, "Numerical simulation of shock wave structure in nitrogen," Phys. Fluids 19, 068102 (2007).

${ }^{33}$ G. A. Bird, Molecular Gas Dynamics and the Direct Simulation of Gas Flows (Oxford University Press, Oxford, 1994).

${ }^{34}$ E. Rathakrishnan, Gas Dynamics (Prentice-Hall of India, New Delhi, 2006).

${ }^{35}$ I. D. Boyd, G. Chen, and G. V. Candler, "Predicting failure of the continuum fluid equations in transitional hypersonic flows," Phys. Fluids 7, 210 (1995).

${ }^{36}$ M. J. Zucrow and J. D. Hoffman, Gas Dynamics (Wiley, New York, 1976), Vol. I.

${ }^{37}$ H. Rabitz and S. H. Lam, "Rotational energy relaxation in molecular hydrogen," J. Chem. Phys. 63, 3532 (1975).

${ }^{38}$ I. D. Boyd, "Rotational-translational energy transfer in rarefied nonequilibrium flows," Phys. Fluids A 2, 447 (1990).

${ }^{39}$ A. E. Belikov, "Rotational relaxation in CO free jets," Mol. Phys. 98, 343 (2000).

${ }^{40}$ J. G. Parker, "Rotational and vibrational relaxation in diatomic gases," Phys. Fluids 2, 449 (1959).

${ }^{41}$ E. A. Mason and L. Monchick, "Heat conductivity of polyatomic and polar gases," J. Chem. Phys. 36, 1622 (1962).

${ }^{42}$ V. M. Zhdanov, Transport Processes in Multicomponent Plasma (Taylor \& Francis, London, 2002).

${ }^{43}$ T. Hill, An Introduction to Statistical Thermodynamics (Dover, New York, 1986).

${ }^{44}$ R. A. Alberty, Physical Chemistry, 7th ed. (Wiley, New York, 1987).

${ }^{45}$ G. Herzberg, Molecular Spectra and Molecular Structure (Krieger Publishing Company, Malabar, FL, 1991), Vol. II.

${ }^{46} \mathrm{E}$. Bich, J. Millat, and E. Vogel, "The viscosity and thermal conductivity of pure monatomic gases from their normal boiling point up to $5000 \mathrm{~K}$ in the limit of zero density and at $0.101325 \mathrm{MPa}$," J. Phys. Chem. Ref. Data 19, 1289 (1990).

${ }^{47}$ T. L. Mazely, G. H. Roehrig, and M. A. Smith, "Free jet expansions of binary atomic mixtures: A method of moments solution of the Boltzmann equation," J. Chem. Phys. 103, 8638 (1995).

${ }^{48}$ A. Ramos, G. Tejeda, J. M. Fernández, and S. Montero, "Nonequilibrium processes in supersonic jets of $\mathrm{N}_{2}, \mathrm{H}_{2}$, and $\mathrm{N}_{2}+\mathrm{H}_{2}$ mixtures: (I) Zone of silence," J. Phys. Chem. A 113, 8506 (2009).

${ }^{49} 1 \mathrm{u} \equiv 1 \mathrm{Da} \equiv m_{a}\left({ }^{12} \mathrm{C}\right) / 12$.

${ }^{50}$ C. S. Wang Chang, G. E. Uhlenbeck, and J. de Boer, in Studies in Statistical Mechanics, edited by J. de Boer and G. E. Uhlenbeck (North-Holland, Amsterdam, 1964), Vol. 2.

${ }^{51}$ L. Waldmann, "Transporterscheinungen in Gasen von mittlerem Druck," in Handbuch der Physik, edited by S. Flügge (Berlin, Springer, 1958), Vol. XII, p. 295.

${ }^{52}$ W. E. Köhler and J. Schaefer, "Theoretical studies of $\mathrm{H}_{2}-\mathrm{H}_{2}$ collisions. V. Ab initio calculations of relaxation phenomena in parahydrogen gas," J. Chem. Phys. 78, 6602 (1983).

${ }^{53}$ F. R. W. McCourt, J. J. M. Beenakker, W. E. Köhler, and I. Kuscer, Nonequilibrium Phenomena in Polyatomic Gases (Oxford Science Publications, 1990 and 1991), Vols. 1 and 2.

${ }^{54}$ R. F. Snider, "Relaxation and transport in molecular systems in the gas phase," Int. Rev. Phys. Chem. 17, 185 (1998). 